\title{
Numerical Simulation of Flow over a Rough Bed
}

\author{
K. M. Singh ${ }^{1}$; N. D. Sandham²; and J. J. R. Williams ${ }^{3}$
}

\begin{abstract}
This paper presents results of a direct numerical simulation (DNS) of turbulent flow over the rough bed of an open channel. We consider a hexagonal arrangement of spheres on the channel bed. The depth of flow has been taken as four times the diameter of the spheres and the Reynolds number has been chosen so that the roughness Reynolds number is greater than 70, thus ensuring a fully rough flow. A parallel code based on finite difference, domain decomposition, and multigrid methods has been used for the DNS. Computed results are compared with available experimental data. We report the first- and second-order statistics, variation of lift/drag and exchange coefficients. Good agreement with experimental results is seen for the mean velocity, turbulence intensities, and Reynolds stress. Further, the DNS results provide accurate quantitative statistics for rough bed flow. Detailed analysis of the DNS data confirms the streaky nature of the flow near the effective bed and the existence of a hierarchy of vortices aligned with the streamwise direction, and supports the wall similarity hypothesis. The computed exchange coefficients indicate a large degree of mixing between the fluid trapped below the midplane of the roughness elements and that above it.
\end{abstract}

DOI: 10.1061/(ASCE)0733-9429(2007)133:4(386)

CE Database subject headings: Turbulence; Flow simulation; Channel flow; Bed roughness; Sediment.

\section{Introduction}

The turbulent flow of a fluid over a rough surface is relatively less well understood than that over smooth surfaces. Its practical applications are, however, very important in geophysical as well as engineering applications. For example, flow in natural rivers and flow over a bank of tubes in heat exchange equipment can both be classified as turbulent flow over rough surface. Also, in the case of atmospheric flow, the ground surface is almost always rough. Hence, a clear understanding of such flows is crucial in the accurate modeling of sediment transport, dispersion of pollutants, and heat transfer. In the past few decades, significant experimental work has been carried out on measuring the flow structures that occur over rough surfaces. Raupach et al. (1991) provide a good review of atmospheric and laboratory rough wall studies, and should be referred to for an extensive list of the relevant work. Some of the prominent experimental investigations of the rough bed channel flow include Grass (1971), Nezu (1977), Grass et al. (1991), Grass and Mansour-Tehrani (1996), and Defina (1996). These investigations show that the distinctive pattern of lowvelocity streaks observed for turbulent flow over smooth surfaces is also a characteristic of the near-wall flow over rough bound-

\footnotetext{
${ }^{1}$ Assistant Professor, Dept. of Mechanical and Industrial Engineering, IIT-Roorkee, Roorkee 247667, India. E-mail: singhfme@iitr.ernet.in

${ }^{2}$ Professor, School of Engineering Sciences, Aeronautics and Astronautics, Univ. of Southampton, Southampton SO17 1BJ, U.K. E-mail: n.sandham@soton.ac.uk

${ }^{3}$ Professor, Dept. of Engineering, Queen Mary, Univ. of London, Mile End Rd., London E1 4NS, U.K. E-mail: J.J.R.Williams@qmul.ac.uk

Note. Discussion open until September 1, 2007. Separate discussions must be submitted for individual papers. To extend the closing date by one month, a written request must be filed with the ASCE Managing Editor. The manuscript for this paper was submitted for review and possible publication on September 20, 2004; approved on September 14, 2006. This paper is part of the Journal of Hydraulic Engineering, Vol. 133, No. 4, April 1, 2007. (CASCE, ISSN 0733-9429/2007/4-386-398/ $\$ 25.00$.
}

aries. Some gross features of the flow, e.g., variation of the mean streamwise velocity, rms fluctuations, and Reynolds stresses, are also similar. There is, however, considerable scatter in the available experimental data, especially near the rough bed, presumably due to difficulties in measurements. Hence, much remains to be done to obtain an accurate understanding of the flow behavior, and to quantify the size of coherent structures and turbulent quantities. A direct numerical simulation (DNS) of such flows should be able to provide an accurate picture, and is the objective of the present work.

DNS provides an ideal approach for the solution of timedependent turbulent flows. In DNS, the turbulent motions are resolved down to the Kolmogorov scales. There are no turbulence models involved as in Reynolds averaged Navier-Stokes models or large-eddy simulation (LES). The only downside is that computational demands become excessive at high Reynolds numbers. Beginning with a $32^{3}$ simulation of isotropic turbulence by Orszag and Patterson (1972), use of DNS has become increasingly widespread in the turbulence research with the advances in computing technology. Moin and Mahesh (1998) provide a review of DNS as a research tool covering its various applications, and its impact on turbulence modeling and understanding the structure of the turbulent flows. The computational requirements of DNS have restricted its applications to low Reynolds number flows in relatively simple geometry. For example, let us consider the simulation of channel flows. The first DNS of fully developed channel flow was performed by Kim et al. (1987). Since then, it has been applied to channel flows of increasing physical and geometrical complexities, including flows with free surface (Lam and Banerjee 1992), transpiration (Sumitani and Kasagi 1995), riblets (Choi et al. 1993), etc. Recently, DNS has also been applied to the turbulent flows over two-dimensional roughness elements (Miyake et al. 2000; Leonardi et al. 2003; Ashrafian et al. 2004). The present study and a similar one by Bhaganagar and Kim (2002) represent the DNS of the fully rough flow over three-dimensional (3D) roughness elements.

Let us note that a large variety of rough surfaces are encoun- 


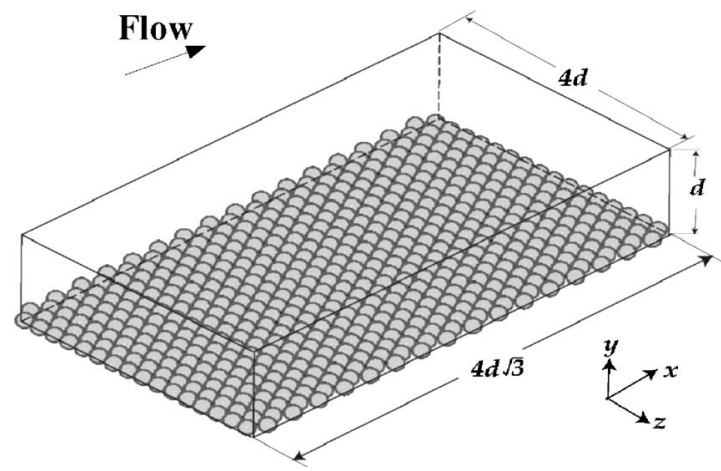

Fig. 1. Channel geometry (computational domain)

tered in the geophysical applications: From plant canopies, gravel beds (in natural rivers and channels) to man-made structures (buildings in a city center in atmospheric pollution studies). We consider a rough bed which represents an idealized model of gravel beds. To simplify the geometric modeling, irregular sized pebbles on the river bed are modeled as spheres of uniform size. For representing the packed nature of the natural bed, we consider a closely packed hexagonal arrangement of spheres of diameter $k$ on the bed. This idealization of the geometry has been partly motivated by the availability of experimental data for flow over a packed bed of spheres (Grass et al. 1991; Grass and MansourTehrani 1996; Defina 1996). The depth of the flow, $d$, is taken to be four times the diameter of the spheres on the bed, and the Reynolds number is chosen to ensure a fully rough flow regime. Based on the data obtained from the DNS, we analyze the features of the flow and compare with published experimental data. We report the first- and second-order statistics, and variation of lift/drag and exchange coefficients (which would be of great relevance for sediment and pollutant transport).

We start with the mathematical description of the problem and computational aspects. We then present statistical features of the flow followed by the effect of roughness elements on flow structures and streak spacing, details of variation of lift/drag and exchange coefficients, and concluding remarks.

\section{Problem Definition}

\section{Channel Geometry and Governing Equations}

We consider fully developed turbulent flow in an open channel having a rough bed consisting of a densely packed array of spheres arranged in a hexagonal lattice. The depth of the flow, $d$, is set at four times the diameter of the spheres, $k$. Fig. 1 gives a schematic representation of a section of the channel used as the computational domain. We choose a Cartesian coordinate system with axes $x, y, z$ (also denoted by $x_{i}, i=1,2,3$ ) pointing in the streamwise, vertical, and spanwise directions, respectively. The origin is located at the bed of the channel, and the incompressible turbulent flow is governed by the Navier-Stokes equation

$$
\frac{\partial u_{i}}{\partial t}+\frac{\partial\left(u_{i} u_{j}\right)}{\partial x_{j}}=-\frac{1}{\rho} \frac{\partial p}{\partial x_{i}}+v \frac{\partial^{2} u_{i}}{\partial x_{j} \partial x_{j}}+b_{i}
$$

and the continuity equation
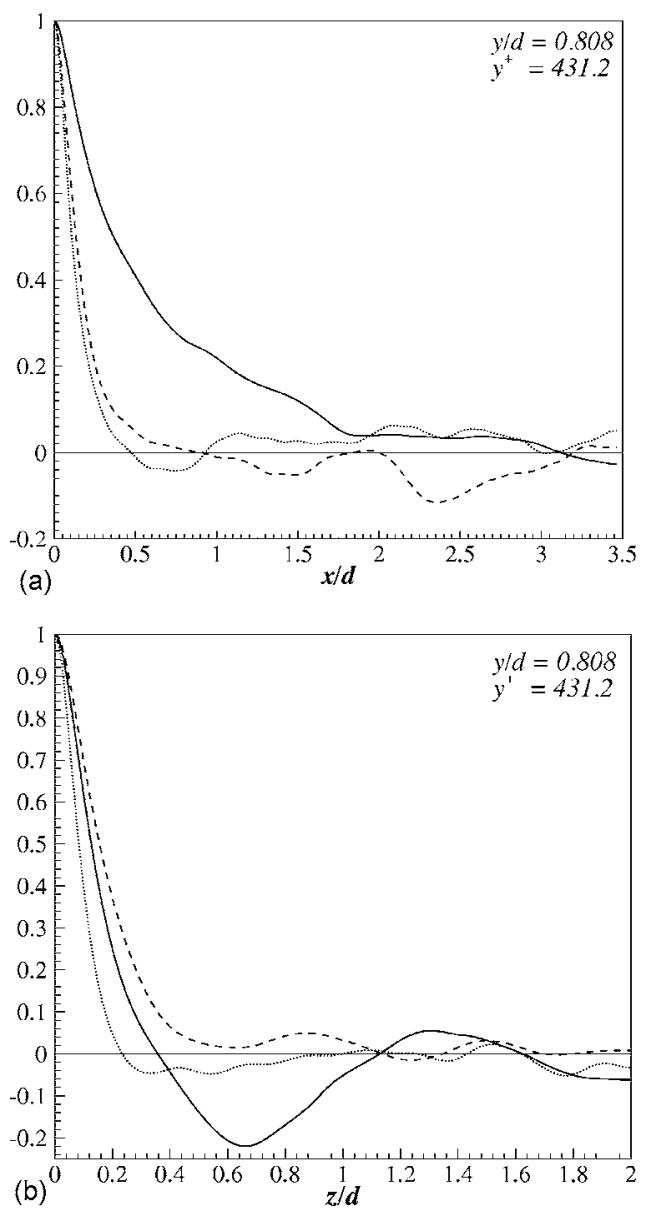

Fig. 2. Two-point correlations: Solid line: $R_{u u}$; dotted line: $R_{v v}$; and dashed line: $R_{w w}$. (a) Streamwise separations; (b) spanwise separations.

$$
\frac{\partial u_{i}}{\partial x_{i}}=0
$$

In the preceding equations, $u_{i}=$ velocity components; $t=$ time; $p=$ pressure; $\quad \rho=$ density; $\quad \nu=$ kinematic viscosity; $\quad$ and $b_{i}=$ components of the body force.

\section{Boundary Conditions and Computational Domain}

The fully developed turbulent channel flow is homogeneous in the streamwise and spanwise directions, hence we can employ periodic boundary conditions in these directions if the computational domain is big enough to include the largest eddies. Based on smooth-bed channel flow DNS (Kim et al. 1987; Lam and Banerjee 1992), we have used a box size of $4 \sqrt{3} d \times 4 d \times d$ (streamwise $\times$ spanwise $\times$ depth) $($ Fig. 1). Here, the factor $\sqrt{3}$ arises from the staggered hexagonal lattice of the spheres on the bed. Since two-point correlations in Fig. 2 are almost zero at the box extremities, the computational domain is sufficiently large in both homogeneous directions. Hence, the use of periodic boundary conditions is fully justified.

No-slip boundary conditions have been used on the bed and sphere surfaces, and a stress-free hard lid was used for the top surface as any surface deformations are likely to be negligible owing to the fact that flow in open channels is due to very shallow slopes. 
In this paper, we adopt the following standard notation: (1) the superscript + indicates a nondimensional quantity scaled by the wall variables, i.e., the kinematic viscosity $v$ and the bed-shear velocity $u_{\tau}$, and (2) an overbar indicates an average over $x, z$, and $t$, and a prime indicates a perturbation from this average.

\section{Computational Aspects}

\section{Numerical Techniques}

The Navier-Stokes equation (1) together with the continuity equation (2) are solved as an initial boundary value problem employing a second-order finite-difference method applied to a staggered grid for spatial discretization. A second-order AdamsBashforth method has been used for time integration, and the Poisson equation for pressure at each time step is solved using a fictitious domain multigrid preconditioner and conjugate gradient method. These numerical techniques have been implemented in a parallel complex geometry DNS-LES code, CgLes, written using $\mathrm{C} / \mathrm{C}++$ and MPI message passing library. Details of the discretization and domain decomposition methods can be found in Thomas and Williams (1995) and Singh and Williams (2005). The computations have been run on the Cray T3E at the University of Manchester, U.K.

\section{Simulation Parameters}

Let $k_{s}$ denote the equivalent sand roughness. The roughness Reynolds number $k_{s}^{+}$defined as

$$
k_{s}^{+}=k_{s} u_{\tau} / v
$$

should be greater than 70 to ensure a fully rough flow. For an appropriate choice of flow parameters, we need to obtain an initial estimate of the equivalent sand roughness $k_{s}$. Experimental data on flow over spherical roughness elements by Defina (1996), and Grass and Mansour-Tehrani (1996) indicate the range of $k_{s} / k$ to be $0.67-0.87$. We choose a median value of $k_{s} / k=0.75$ as our initial estimate, and set the flow Reynolds number $\mathrm{R}^{+}=u_{\tau} d / v$ $=533$ corresponding to the roughness Reynolds number $k_{s}^{+}=100$. This choice of Reynolds number should ensure that the actual $k_{s}^{+}$ would be greater than 70 .

For the finite-difference discretization, we have used a Cartesian grid of resolution $1024 \times 512 \times 128$ in the streamwise, spanwise, and vertical directions, respectively. This gives us approximately $2.7 \times 10^{8}$ degrees of freedom, and grid sizes of $0.87 d / 128, d / 128$, and $d / 128$ in the streamwise, spanwise, and vertical directions. Based on the average dissipation rate per unit mass over the computational domain, the Kolmogorov length scale $\eta^{+}$is estimated to be about 3.0 wall units. The grid sizes (in wall units) $\Delta x^{+}, \Delta y^{+}$, and $\Delta z^{+}$are $3.6,4.2$, and 4.2, respectively, which are very close to the Kolmogorov length scale $\eta^{+} \approx 3.0$. Sufficiency of the grid resolution has also been verified from the wave number spectra (in the periodic directions) and dissipation length scales-Kolmogorov microscale $\eta$ as well as directional dissipation scales $\eta_{i j}$ of Manhart (2000) at each vertical grid plane. Hence, no subgrid mode is used in the computations. The following list summarizes the simulation parameters for the DNS:

- Computational box: $4 \sqrt{3} d \times 4 d \times d$;

- Height of roughness elements: $k=d / 4$;

- Reynolds number: $\mathrm{R}^{+}=u_{\tau} d / \nu=533$;

- Body force: $b_{x}=u_{\tau}^{2} / d, b_{y}=0, b_{z}=0$;

- DNS grid: $1024 \times 512 \times 128$;
- Grid resolution: $\Delta x^{+}=3.6, \Delta y^{+}=\Delta z^{+}=4.2$;

- Kolmogorov length scale: $\eta^{+} \approx 3.0$; and

- Target roughness Reynolds number: $k_{s}^{+}>70$

\section{Initial Condition and Computational Runs}

In order to keep the computational requirements to the minimum, we first carry out a LES at one half the above-mentioned resolution. The LES is run for around $30 T$ (where $T=d / u_{\tau}$ is the largeeddy turnover time) to obtain a fully developed turbulent field. The statistically steady (fully developed turbulent) state is identified by a linear equilibrium profile of the total shear stress, $\overline{-u^{\prime} v^{\prime}}+v \partial \bar{u} / \partial y$, away from the roughness elements. This fully developed turbulent flow field is interpolated onto the DNS grid to yield the initial conditions for the DNS. Time integration is performed on the fine grid for several $T$ until the effect of the initial conditions has vanished and the velocity field reaches the fully developed state as indicated by the profile of total shear stress. To obtain statistics of second-order quantities, the time integration is continued further for several $T$, and the statistical quantities are obtained by averaging in time as well as over the horizontal planes. Fine resolution runs were carried out using 256 processors on a Cray $\mathrm{T} 3 \mathrm{E}$ and the total number of processor hours used for the complete simulation is estimated to be 200,000. Such an amount of computer time is obviously very large and precludes the use of a bigger box or greater resolution. It is worthwhile noting that a doubling of resolution would increase run times by a factor of 16 for the same time step ratio.

\section{Statistical Features of Rough Bed Flow}

The accuracy and physical correctness of the DNS results can be ascertained only by comparison with the available experimental results. Quantitative experimental data from Grass (1971), Nezu (1977), Grass et al. (1991), and Defina (1996) are available in the form of the first- and second-order statistics, and these are compared with the temporal and plane averaged statistics obtained from the DNS. Let us note that in view of the difficulties involved in accurate measurements near the top of the roughness elements, comparison would be meaningful only above the so-called roughness layer (Raupach et al. 1991). We first determine the effective position of the bed and equivalent sand roughness, and then present the comparative analysis of the mean velocity, turbulence intensities, Reynolds stress, and related measures.

\section{Effective Position of Bed and Equivalent Sand Roughness}

The statistical description of turbulent flow over a rough bed can be characterized by two parameters, namely (1) the effective location of the bed, $y_{b}$; and (2) a roughness length, $y_{0}$, both of which are determined from the flow statistics. The roughness length $y_{0}$ is related to a measure of the size of the roughness elements $k_{*}$ by

$$
y_{0}=k_{*} / A
$$

where $A=$ constant. It is a matter of debate whether $k_{*}$ should be equal to the mean height of the roughness elements, $k$, or to the so-called equivalent sand roughness $k_{s}$. Recent experimental literature on open channel flows favors the latter option, and we adopt it in this paper for comparison with these experimental 
Table 1. Mean Flow Variables Obtained from DNS

\begin{tabular}{lc}
\hline Flow variable & Related Reynolds number \\
\hline Equivalent sand roughness, $k_{s}=0.77 k$ & $k_{s}^{+}=k_{s} u_{\tau} / \nu=102$ \\
Effective flow depth, $h=d-y_{b}=3.19 k$ & $\mathrm{R}_{h}^{+}=u_{\tau} h / \nu \approx 425$ \\
Bulk mean velocity, $U_{b}=7.32 u_{\tau}$ & $\mathrm{R}_{b}=U_{b} h / \nu \approx 3112$ \\
Mean velocity of the top surface, $U_{c}=11.6 u_{\tau}$ & $\mathrm{R}_{c}=U_{c} h / \nu \approx 4955$ \\
\hline
\end{tabular}

results. For fully rough channel flows, $A \approx 30$ (Raupach et al. 1991; Grass et al. 1991).

There are several techniques for determining these parameters (Raupach et al. 1991), each yielding a different set of values. In the channel flow literature, both of these parameters are normally estimated from the mean velocity distribution in the region where it coincides with the log-law of the wall region given by

$$
U^{+}(y)=\frac{1}{\kappa} \log \left[\frac{\left(y-y_{b}\right)}{y_{0}}\right]=\frac{1}{\kappa} \log \left[A \frac{\left(y-y_{b}\right)}{k_{s}}\right]
$$

where $U^{+}=\bar{u} / u_{\tau}$ and $\kappa=$ von Kármán constant (we have used $y_{0}=k_{s} / A$ ). For example, Grass et al. (1991) determine $y_{b}$ and $k_{s}$ by using a combination of the origin shift method and linear regression to obtain the best-fit straight line in the logarithmic zone. For a fully rough case $\left(k_{s}^{+}=79.2\right)$, they find $y_{b}=0.783 k$ and $k_{s}=0.88 k$. Defina (1996) determines $k_{s}$ by fitting mean velocity profiles to the logarithmic law-of-the-wall, and obtains $k_{s}=0.67 k$. We use a similar approach, and determine these values by solving a two parameter nonlinear curve-fitting problem using the least squares and Newton-Raphson techniques. The minimization procedure yields $y_{b}=0.81 k$ and $k_{s}=0.768 k$ for the best fit of the DNS data with the log-law profile in the logarithmic zone. Thus, the roughness Reynolds number for the present simulation is $k_{s}^{+}=102$ indicating a completely rough flow regime. Next, let us suppose that the effective location of the bed is at a distance $\delta$ below the top of the roughness elements. Nezu and Nakagawa (1993) indicate that the range of $\delta / k$ is about $0.15-0.3$. For the present case, $\delta / k=0.19$, which is close to the lower bound of the suggested range. Further, with the effective location of the bed near the top of the roughness elements, the effective flow depth $h$ is equal to $d-y_{b}$. For comparison with the experimental data, we define a vertical coordinate $Y$ with origin at $y=y_{b}$, i.e., $Y=y-y_{b}$. Table 1 lists these values together with other mean properties computed based on the DNS data.

\section{Mean Velocity}

The mean streamwise velocity profile normalized with the bedshear velocity $u_{\tau}$ is presented in Fig. 3 . We can clearly see the absence of the viscous sublayer and the presence of a retarded fluid layer near the bed $\left(Y^{+}<60\right)$ due to the penetration of the roughness elements into the logarithmic region. Comparison with the experimental results of Grass et al. (1991) and Defina (1996) show that the DNS profile follows the standard pattern of velocity defect increasing with wall roughness.

A better comparison of the DNS result with the experimental data can be made by plotting the mean velocity with respect to the vertical length scaled by the equivalent sand roughness $k_{s}$. Fig. 4 presents the plot of $U^{+}$against $Y / k_{s}$. The mean velocity profile obtained with DNS shows excellent agreement with the experimental results of Defina (1996) and Grass et al. (1991).

The mean flow below the top of the roughness elements is shown in Fig. 5. Some interesting features can be observed in the flow around the roughness elements: The presence of a viscous

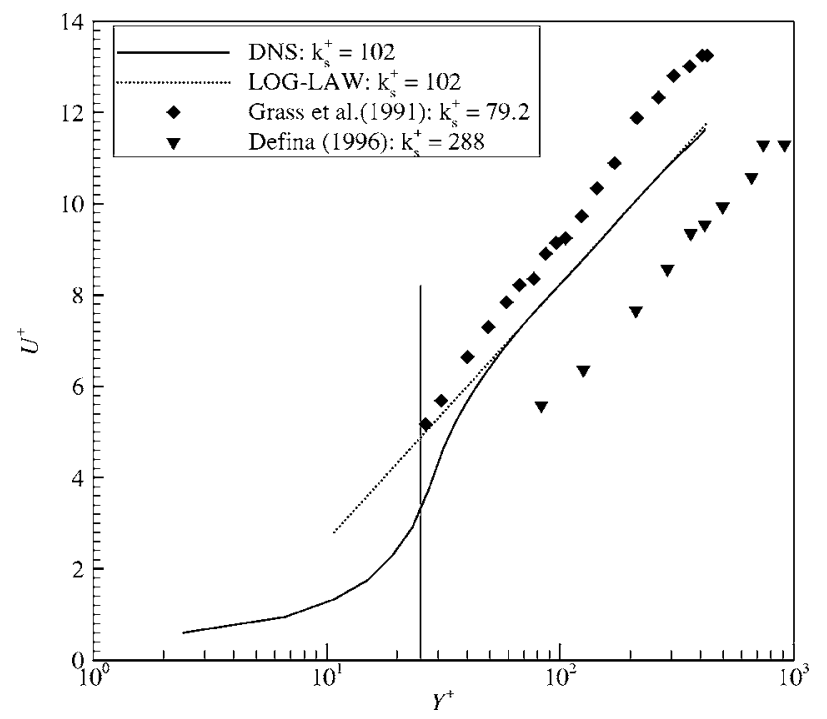

Fig. 3. Mean velocity profiles

sublayer near the bed $\left(y / d<0.06, y^{+}<32\right)$, a fully turbulent layer on top of it, and a mild back flow near the midplane followed by a sharp velocity gradient. Moreover, the fluid flow around the midplane of the roughness elements is turbulent as indicated by the variation of the effective eddy viscosity, $v^{\prime}$, which is defined as

$$
v^{\prime}=-\frac{\overline{u^{\prime} v^{\prime}}}{(\partial \bar{u}) / \partial y}
$$

\section{Turbulence Intensities}

Turbulence intensities normalized by the bed-shear velocity are shown in Fig. 6. We have also included the experimental values from Grass (1971) and Nezu (1977) corresponding to roughness Reynolds numbers of 84.7 and 136, respectively. Since these roughness Reynolds numbers are based on the roughness height $k$, these values actually correspond to $k_{s}^{+} \approx 74.5$ for Grass (1971) and

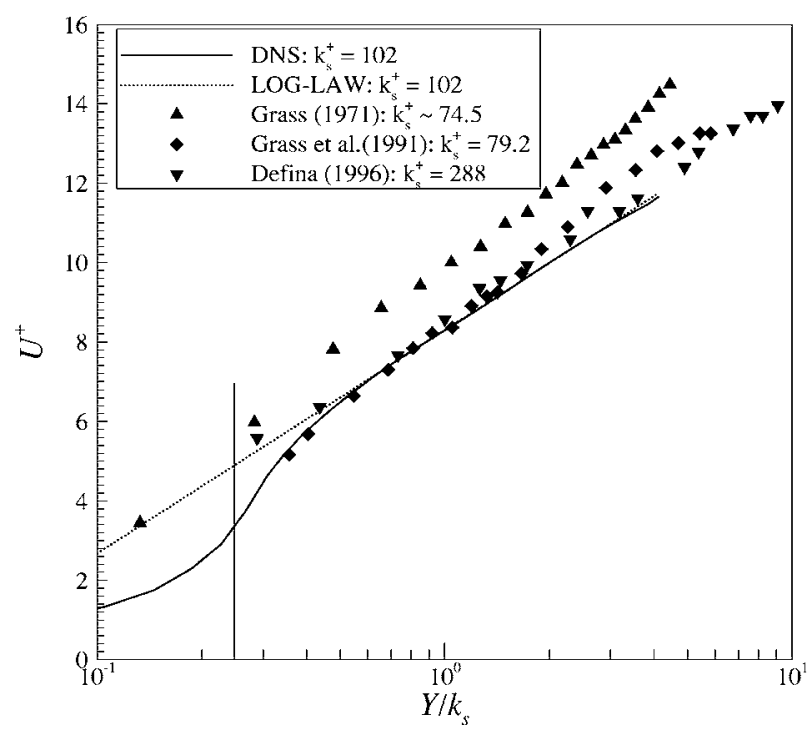

Fig. 4. Mean velocity profiles plotted against $Y / k_{s}$ 


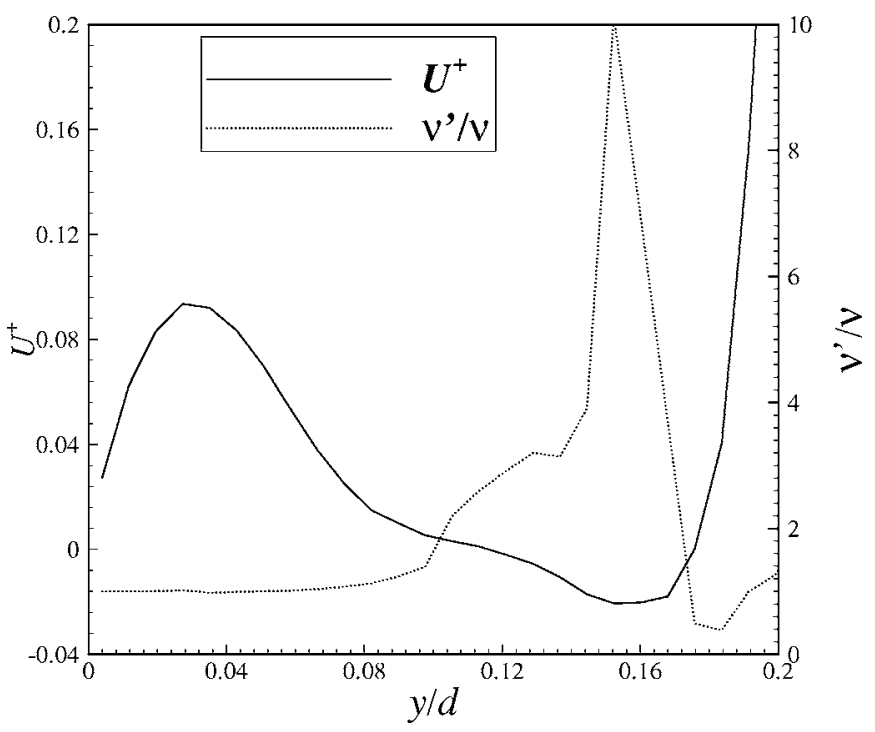

Fig. 5. Mean velocity and scaled effective viscosity near bed

$k_{s}^{+} \approx 100$ for Nezu (1977). Thus, data from Grass (1971) correspond to a less rough bed, whereas those of Nezu (1977) correspond to a roughness condition very similar to that of the present DNS. The computed values are lower than the experimental values of Grass (1971), but show a fairly good agreement with the experimental data of Nezu (1977) everywhere except near the effective bed. Near the top of the roughness elements, the computed values are much lower than the measured values and any differences could be due to possible difficulty in obtaining reliable measurements in these locations. The overall trend in the turbulence intensities is very similar to that observed by Kim et al. (1987) for the smooth-bed channel flow. At the same time, the peak value of $u_{\mathrm{rms}} / u_{\tau}$ is 1.83 , which is close to the experimentally observed value 1.9 found by Nezu (1977). Two peaks can be seen in the profile for the spanwise fluctuations: One

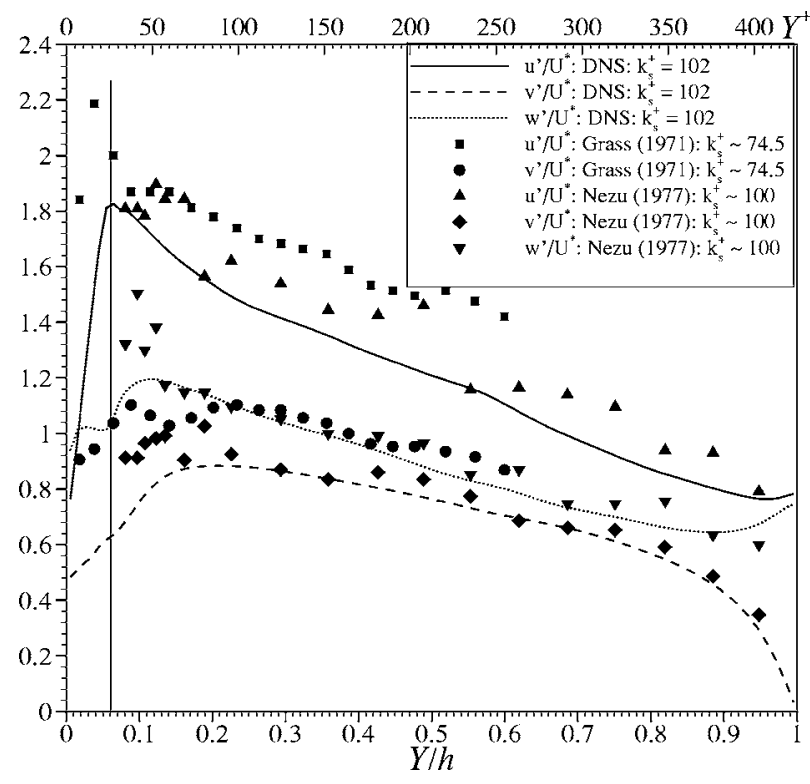

Fig. 6. Turbulent intensities. $u^{\prime}, v^{\prime}$, and $w^{\prime}$ represent rms velocities and $U^{*} \equiv u_{\tau}$. Thin vertical line denotes the top of the roughness elements.

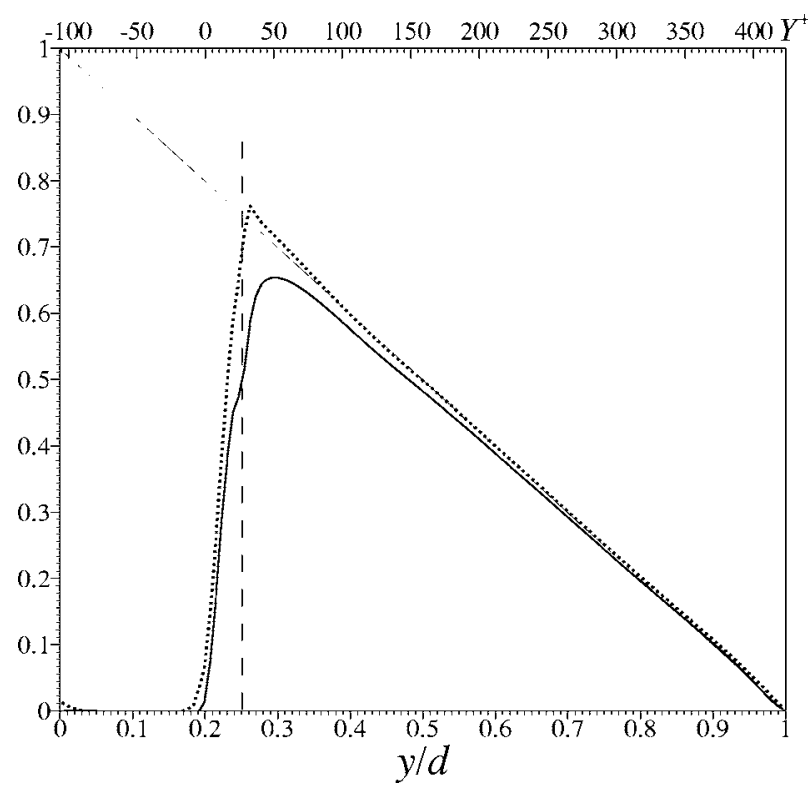

Fig. 7. Reynolds shear stress normalized by the bed-shear velocity: solid line: $-\overline{u^{\prime} v^{\prime}} / u_{\tau}^{2}$; normalized total shear stress: dotted line $\left(-\overline{u^{\prime} v^{\prime}}+v \partial \bar{u} / \partial y\right) / u_{\tau}^{2}$. Thin solid line represents the profile of total shear stress for fully developed smooth wall flow. Dashed vertical line denotes the top of the roughness elements.

below $\left(y / d=0.22, Y^{+}=11\right)$ and one above $\left(y / d=0.29, Y^{+}=48\right)$ the top of the roughness elements. This feature marks the difference in the near-bed profiles of turbulent fluctuations between the smooth and rough bed flows.

\section{Reynolds Stress}

The Reynolds shear stress normalized by the bed-shear stress is shown in Fig. 7 together with the total shear stress. In fully developed channel flow, the profile of the total shear stress should be a straight line away from roughness elements (indicated by the thin straight line). The absence of any significant deviations in the total shear stress profile from the straight line confirms that the turbulent flow is indeed fully developed. The peak values of the Reynolds shear stress and the total shear stress occur at $y / d=0.29\left(Y^{+} \approx 48\right)$ and $y / d=0.26\left(Y^{+} \approx 34\right)$, respectively.

Next, let us consider the correlation coefficient of the Reynolds stress defined as

$$
R=-\overline{u^{\prime} v^{\prime}} /\left(u_{\mathrm{rms}} v_{\mathrm{rms}}\right)
$$

Based on the experimental data for channel flows, boundary layers, and pipe flows (fully smooth to fully rough), Nezu and Nakagawa (1993) note that $R$ should increase monotonically with $y / h$ near the bed, decrease monotonically in the free surface region, and remain nearly constant (about $0.4-0.5$ ) in the intermediate region irrespective of the properties of the mean flow and the wall roughness. Fig. 8 shows the distribution of $R$ with the normalized flow depth $Y / h$. The DNS values agree fairly well with the preceding observation of Nezu and Nakagawa (1993) and the experimental data of Nezu (1977) in the bottom half of the channel. The DNS profile for $R$ for this rough bed flow is very similar to that of the smooth bed channel flow (Kim et al. 1987) with the exception that we see two local peaks near the effective bed as opposed to one observed near the smooth bed. The first peak occurs at $Y^{+} \approx 15$, which is slightly above the location of the 


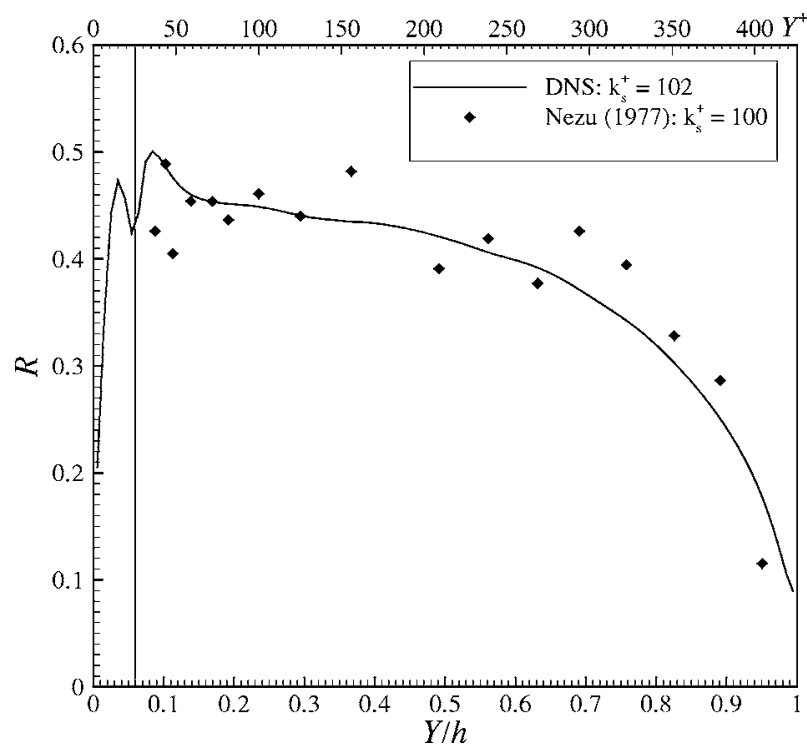

Fig. 8. Correlation $R$ of Reynolds shear stress, $\left(h=d-y_{b}\right)$

first peak for spanwise velocity fluctuations, $w_{\text {rms }}\left(Y^{+} \approx 11\right)$. The second peak occurs at $Y^{+} \approx 35$, which is slightly above the peak location for streamwise turbulent fluctuations, $u_{\mathrm{rms}}\left(Y^{+} \approx 27\right)$, and coincides with the location of the maximum total shear stress.

The coefficient $R_{k}$ defined by

$$
R_{k}=-\overline{u^{\prime} v^{\prime}} /(2 K)
$$

is an indicator of the correlation between the Reynolds stress and the turbulent kinetic energy $K=\left(\overline{u^{\prime 2}}+\overline{v^{\prime 2}}+\overline{w^{\prime 2}}\right) / 2$. The values of $R_{k}$ (Fig. 9) are somewhat higher than the experimental values of Nezu (1977) near the roughness elements $(y / h<0.3)$, but show reasonably good agreement elsewhere. The DNS profile of $R_{k}$ shows a nearly constant value $(\approx 0.14)$ in the intermediate region, which is in agreement with the observation of Nezu and Nakagawa (1993) (who indicate that $R_{k}=0.12-0.16$ in the intermediate region).

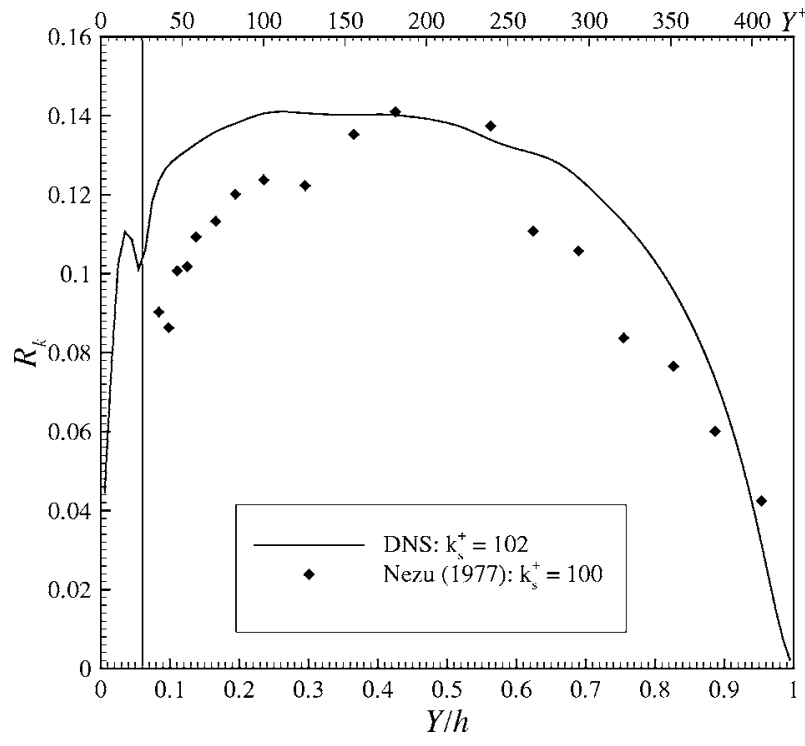

Fig. 9. Ratio of Reynolds shear stress to turbulent kinetic energy, $R_{k}$

\section{Structure of Turbulent Flow over Roughness Elements}

We investigate the flow features responsible for the low-speed streaks, Reynolds stress production, and sediment/scalar transport. In this section, we focus our attention on the flow structures primarily over the roughness layer to investigate the similarity and differences compared with smooth wall flows. The effect these near-wall coherent structures have on scalar/sediment transport will be taken up in the next section.

Turbulent flow in close proximity to the roughness elements is certainly influenced by the roughness type and length scale. However, experimental investigations indicate a decoupling of the flow field from the effect of the roughness elements further away from the roughness layer. Grass et al. (1991) show that the nearwall bursting events characterized by sweeps and ejections which are present above smooth walls are also present in rough wall turbulent flows, and thus, indicate that the coherent structures should also be similar. This apparent similarity of turbulent flow over different surface types has been termed the wall similarity hypothesis by Raupach et al. (1991). We investigate the validity or otherwise of this hypothesis based on the DNS data using two-point correlations, planar snapshots, and quadrant analysis.

\section{Effect of Roughness Length Scale}

The effect of the roughness length scale can be clearly observed in the plot of the two-point correlations shown in Fig. 10 at different $y$ locations. Numerous well-defined peaks in these plots indicate the presence of small-scale disturbances around individual spheres. However, the correlations just a small vertical distance above the top of the spheres in Fig. 11 show a much smoother profile, and thus, indicate the decoupling of the flow field from the influence of the individual roughness elements. These results confirm the observation of Grass and MansourTehrani (1996) on the apparent ability of the rough wall flow to reorder itself very rapidly over a small vertical distance above the roughness elements, and thereby provide a confirmation of the wall similarity hypothesis of Raupach et al. (1991).

\section{Presence of Bursting Events}

To investigate the presence of bursting events responsible for the near-wall coherent flow structures, we have observed the snapshots of the flow field at various streamwise and spanwise planes. We include here the velocity and pressure distributions on a representative vertical plane aligned with the streamwise direction in Fig. 12. The velocity field is in a convected frame of reference moving with the bulk velocity $U_{b}$. We can clearly see characteristic signatures of the bursting events, namely the cross sections through transverse vortices, ejection, and sweep zones (for the sake of illustration, we have marked the ejection by $\mathbf{E}$ and the sweep event by $\mathbf{S}$ in Fig. 12). Occurrence of elongated lowpressure zones, some of which extend close to the top of the channel, indicates the presence of a few large vortical structures presumably formed by the coalescence of smaller vortices originating near the top of the roughness elements. These observations further confirm the similarity of the events in smooth and rough wall flows.

\section{Presence of Low-Speed Streaks}

Distinctive patterns of low-velocity streaks which are observed in smooth walls have also been observed in rough wall flows in 

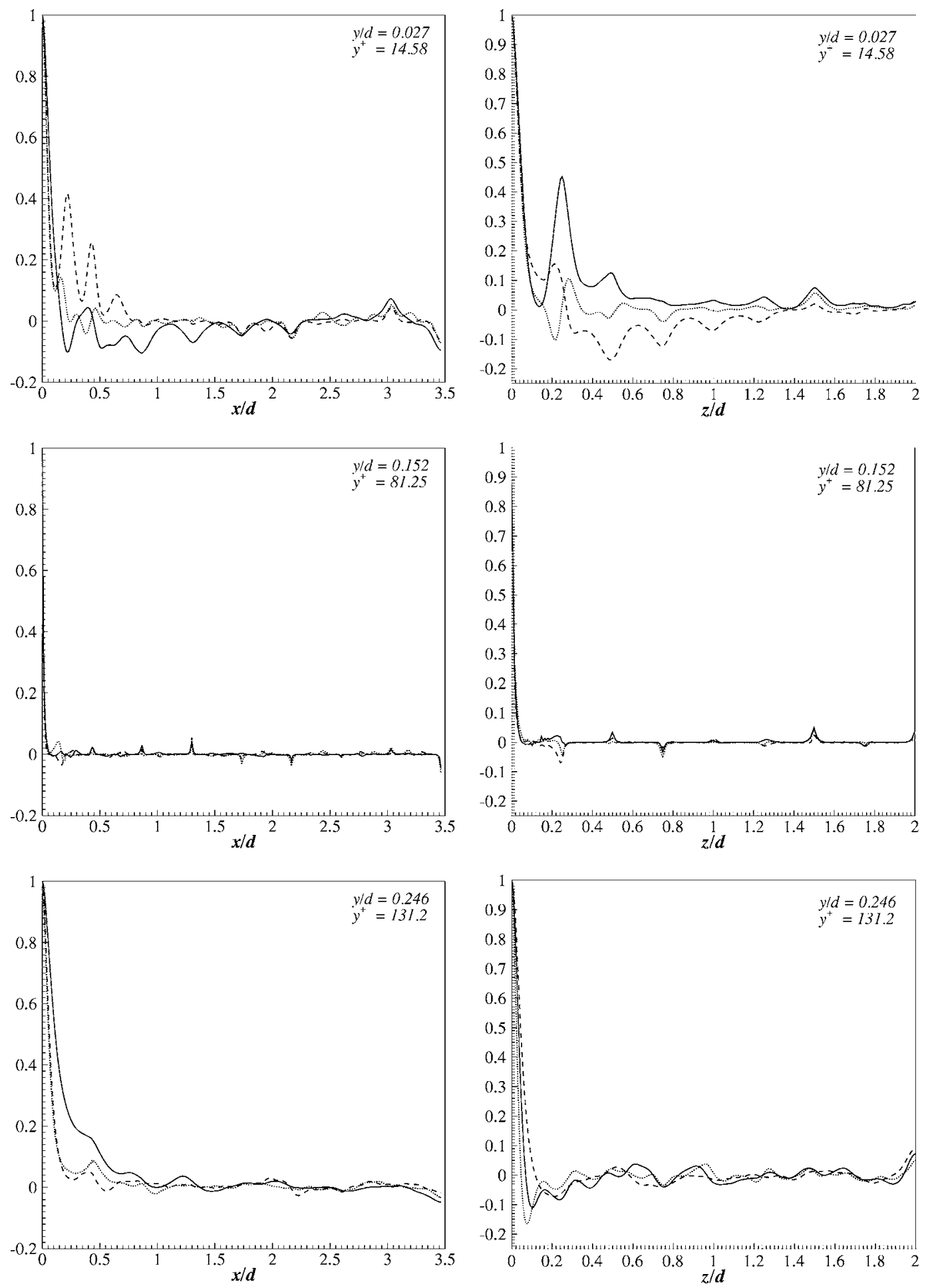

(a) Streamwise separations

(b) Spanwise separations

Fig. 10. Two-point correlations at different $y$ locations near the bed: solid line: $R_{u u}$; dotted line: $R_{v v}$; and dashed line: $R_{w w}$. (a) Streamwise separations; (b) spanwise separations.

experiments by Grass et al. (1991) and Defina (1996). Plots of $u^{\prime}<0$ obtained from the DNS at two different vertical locations are presented in Fig. 13. These plots clearly confirm the presence of elongated low-speed streaks in the rough bed flow. Further, we can observe an increase in average separation between the streaks as we move away from the bed.
We can estimate the mean transverse spacing of the streaks from the plot of two-point correlations. Let $\delta z$ be the distance to the first minimum of the spanwise correlation of streamwise velocity. The mean transverse spacing between the streaks, $\lambda$, is roughly $2 \delta z$. Its variation with the distance from the top of the roughness elements is plotted in Fig. 14. For comparison with the 

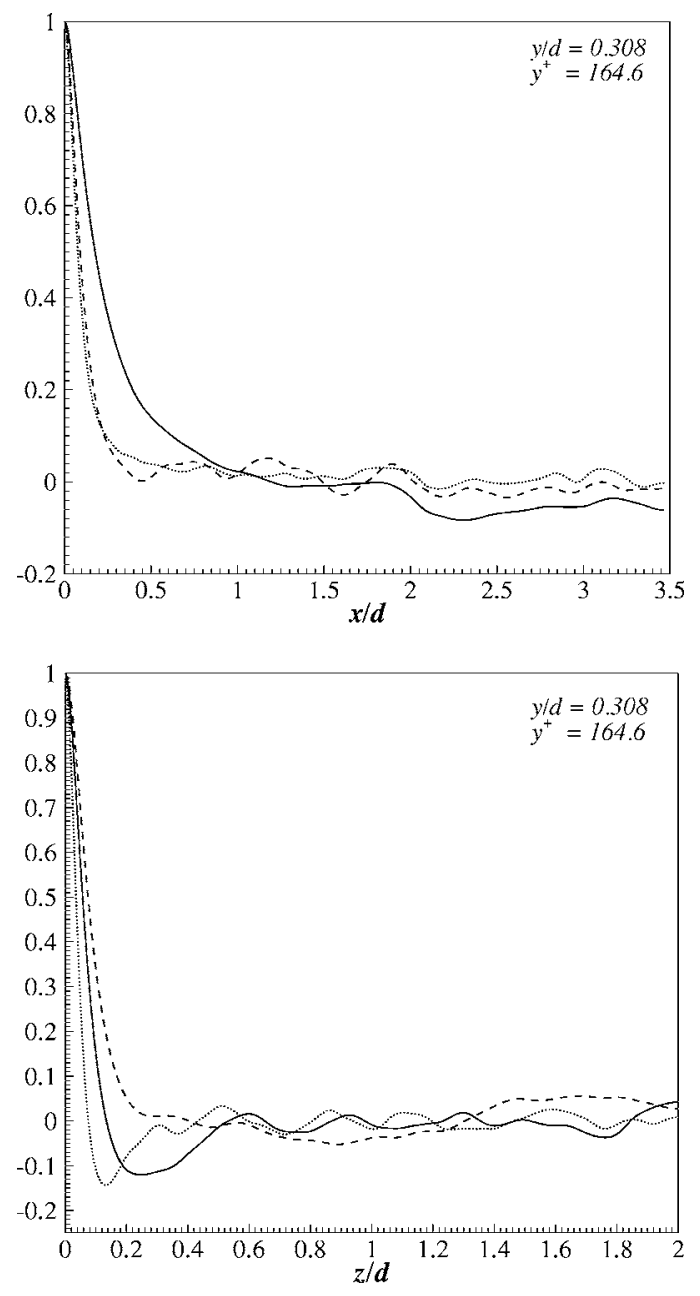

Fig. 11. Two-point correlations at a small vertical distance above the roughness elements: solid line: $R_{u u}$; dotted line: $R_{v v}$; and dashed line: $R_{w w}$. (a) Streamwise separations; (b) spanwise separations.

experimental data of Defina (1996) (identified by S2 and S3 in this plot), we have scaled the spacing $(\lambda)$ as well as the distance from the top of the roughness elements $\left(y_{t}\right)$ by the equivalent sand roughness $k_{s}$. Near the top of the spheres, $\lambda / k_{s} \approx 3.2$, which is close to $\lambda / k_{s} \approx 3.8$ suggested by Grass and Mansour-Tehrani (1996). We can observe that this value is nearly constant near the top of the roughness elements $\left(y_{t} / k_{s}<0.5\right)$, which indicates the presence of a single vortical hierarchy active near the top of the spheres. Further up from the bed, the streak spacing grows, but

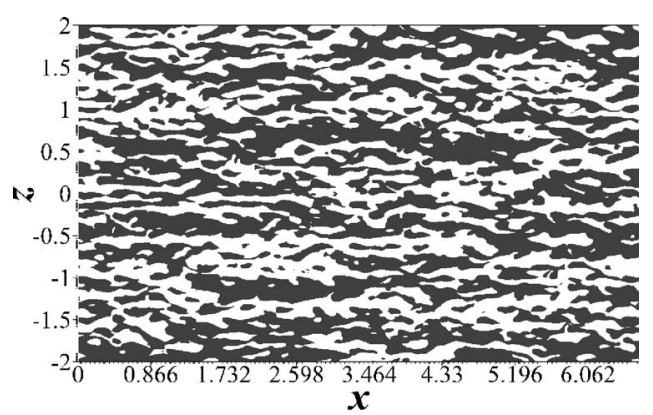

(a)

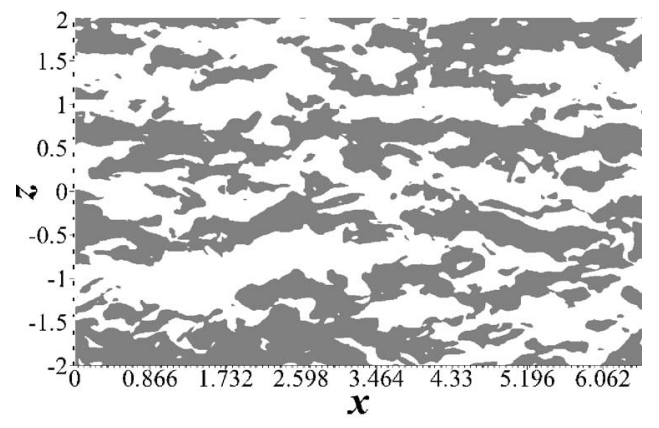

(b)

Fig. 13. Low speed streaks (gray shaded) in the rough-bed flow. (a) $y / d=0.254$; (b) $y / d=0.371$.

the growth pattern is rather nonuniform suggesting the presence of different sets of interlinked vortical hierarchies. A uniform linear growth is seen only near the top surface.

Let us look at the generalized scaling of near wall vortical structures. Following Grass and Mansour-Tehrani (1996), we can treat the fluid layer near the top of the spheres as a reconstituted fluid of enhanced effective viscosity. Near the effective bed (i.e., the estimated velocity origin), the velocity gradient and the effective viscosity, $v^{\prime}$, are not constant. Using the value of streak spacing $\lambda$ at $y=k+k / 3$ and an effective viscosity taken near the top of the roughness elements $\left(v^{\prime}=0.0065\right)$, we obtain $\lambda^{+} \approx 103$. This value is very close to $\lambda^{+} \approx 100$ suggested by Grass and MansourTehrani (1996), and reinforces their suggestion that $\lambda^{+}$has a constant value of 100 irrespective of the wall roughness. This suggestion must be linked to the authors' previous statements on the similarity of rough and smooth wall flow above the wall roughness and viscous sublayer, respectively.

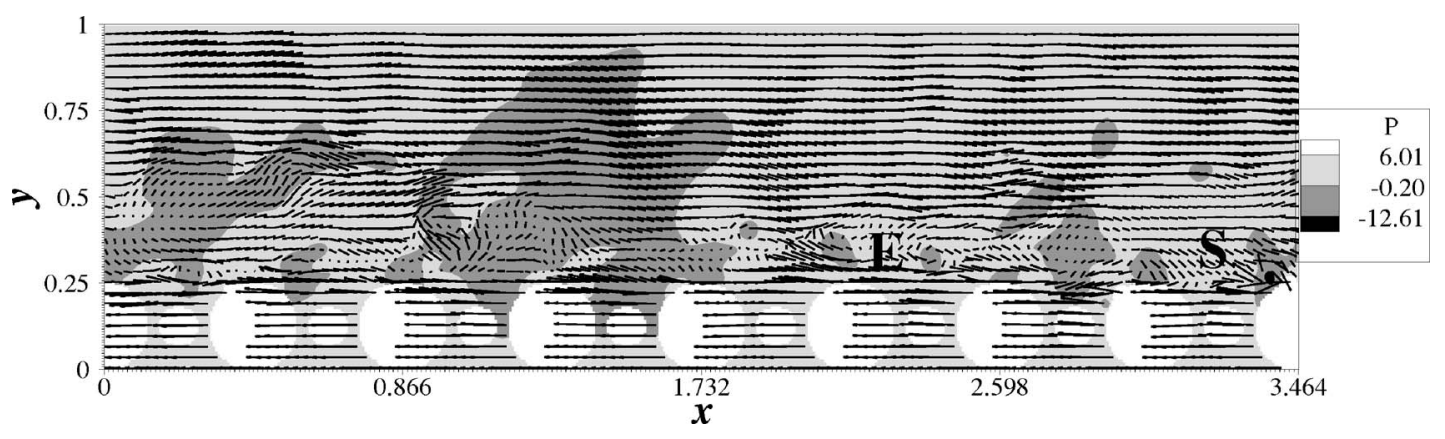

Fig. 12. Velocity vectors in advected frame of reference $\left(u-U_{b}, v\right)$ and contours of pressure on a streamwise-vertical plane $(z / d=0.012)$ 


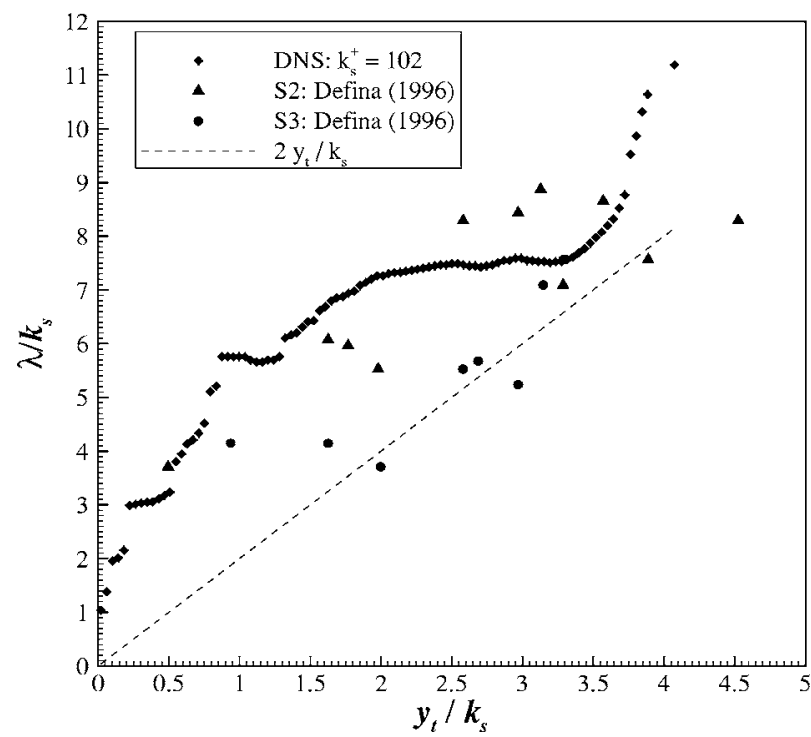

Fig. 14. Transverse spacing of streaks scaled by equivalent sand roughness $k_{s} . y_{t}=$ distance from the top of the roughness elements and the dashed straight line is $\lambda=2 y_{t}$.

\section{Turbulence Production: Quadrant Analysis}

To further compare the turbulence production between smooth and rough wall flows, we employ quadrant analysis in order to obtain detailed information on the contribution of various events to the total turbulence production. The events in the flow are linked to the quadrants in the $\left(u^{\prime}, v^{\prime}\right)$ plane ( $\mathrm{Lu}$ and Wilmarth 1973). The first quadrant $\left(u^{\prime}>0, v^{\prime}>0\right)$ contains outward motion of high speed fluid, also referred to as an outward interaction event. The second quadrant $\left(u^{\prime}<0, v^{\prime}>0\right)$ is associated with the ejection of low speed fluid away from the wall. The third quadrant $\left(u^{\prime}<0, v^{\prime}<0\right)$ is linked with inward interaction events (i.e., motion of low speed fluid toward the bed); whereas the fourth quadrant is associated with the sweep event (i.e., the inrush of high-speed fluid). The even quadrants contribute to positive production whereas the odd quadrants contribute to negative production of the Reynolds shear stress. Fig. 15 presents the contribution to the Reynolds shear stress from each quadrant above the effective bed. We can clearly see the dominance of ejection events (second quadrant) away from top of the roughness elements $\left(Y^{+}\right.$

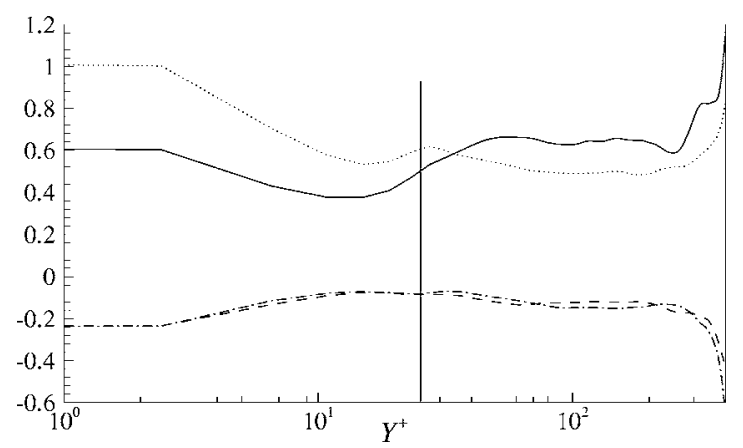

Fig. 15. Contribution to Reynolds shear stress from each quadrant normalized by the local mean Reynolds shear stress: dashed line: first quadrant; solid line: second quadrant; dotted-dashed line: third quadrant; and dotted line: fourth quadrant
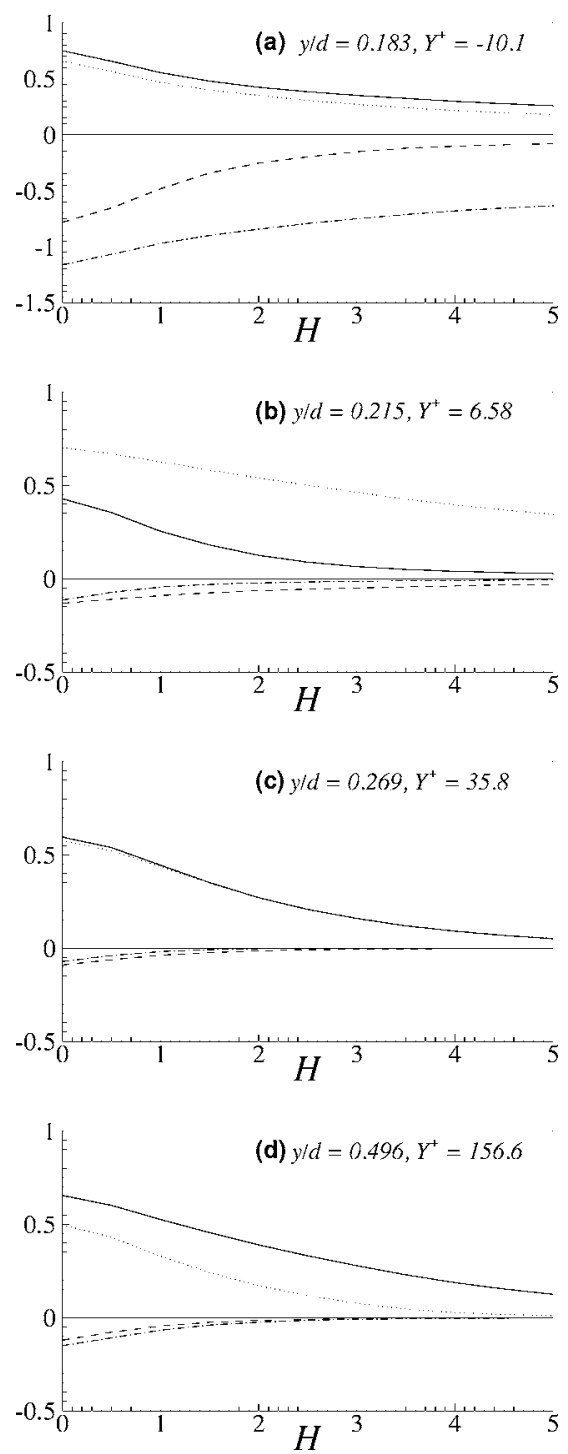

Fig. 16. Fractional contribution to Reynolds shear stress from each quadrant: dashed line: first quadrant; solid line: second quadrant; dotted-dashed line: third quadrant; and dotted line: fourth quadrant

$>36$ ). However, sweep events (fourth quadrant) dominate near the top of the roughness elements (and above the effective bed).

To extract the dominant contributions to the Reynolds stress from each quadrant, Lu and Wilmarth (1973) introduce a threshold level $H$, called the hole size, and thus, divide the $\left(u^{\prime}, v^{\prime}\right)$ plane into five regions as follows:

- ith quadrant: If $\left|u^{\prime} v^{\prime}\right|>H u_{\mathrm{rms}} v_{\mathrm{rms}}$ and the point $\left(u^{\prime}, v^{\prime}\right)$ is in the $i$ th quadrant in the $\left(u^{\prime}, v^{\prime}\right)$ plane.

- Hole region: If $\left|u^{\prime} v^{\prime}\right| \leqslant H u_{\mathrm{rms}} v_{\text {rms }}$.

Fig. 16 presents the fractional contributions from the four quadrants as a function of the hole size at selected vertical locations. Just below the effective bed $(y / d=0.183)$, inward interaction events dominate resulting in negative production of Reynolds shear stress. Above the effective bed, the trend is very similar to that observed for smooth bed channel flow (Kim et al. 1987), i.e., intense $u^{\prime} v^{\prime}$ events $(H>3)$ would always be either from the second or the fourth quadrant. Sweep events (fourth quadrant) dominate near the effective bed [Fig. 16(b)], whereas ejection events (second quadrant) dominate further away [Fig. 16(d)]. Moreover, 


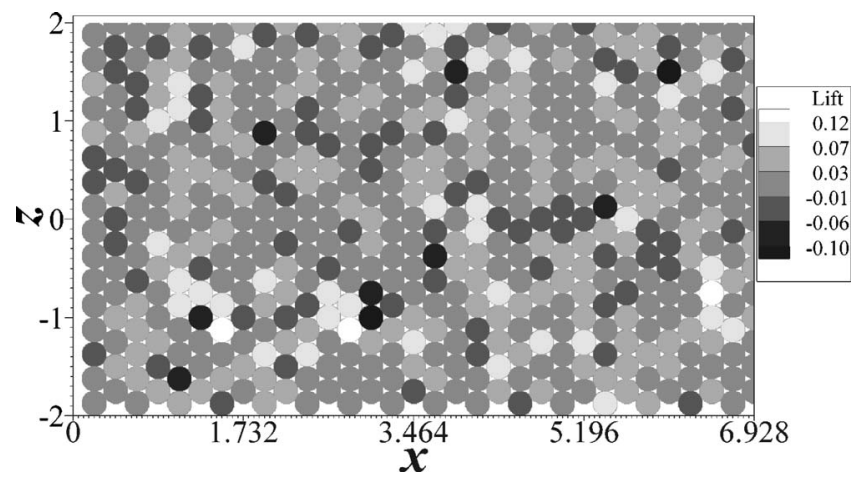

Fig. 17. Distribution of lift on the roughness elements

at the location where contributions from sweep and ejection events are nearly the same $\left(Y^{+} \approx 36\right)$, even their fractional contributions are identical [Fig. 16(c)].

Thus, the DNS results for the rough bed flow show the presence of the imprints of bursting events, low-speed streaks, and Reynolds stress production very similar to that observed over smooth walls. These observations add further support to the wall similarity hypothesis by Raupach et al. (1991), which states that rough-wall and smooth-wall boundary layers at sufficiently high Reynolds numbers are structurally similar (i.e., that organized motions are similar) outside the roughness and viscous sublayers, respectively. The effective viscosity length scale $\lambda^{+} \approx 103$ is in remarkable agreement with the experimental value of Grass and Mansour-Tehrani (1996), and confirms their suggestion that the spacing of low-speed streaks adjacent to the bed scales directly with the length scale/size of the roughness elements regardless of the Reynolds number. This confirmation gives us further confidence to predict that the spread of scalars in different geophysical flows (e.g., the cross wind spacing of fluid ejection zones, lifting and dispersal of pollutants in the air or water bodies) would be of the order of 100 roughness length scales (Grass and MansourTehrani 1996).

\section{Implications for Sediment Transport}

The coherent structures generated by bursting events (ejections and sweeps) provide the driving mechanisms for coarse as well as fine grain sediment transport. Low pressure zones generated by the vortical structures above a roughness element would result in a lift force which would play a significant role in bed-instability and coarse grain sediment transport. On the other hand, fluctuating vertical velocity components induced by ejections and sweeps would be instrumental in the mixing of the fluid layers above and below the midplane of roughness elements, and thereby play the dominant role in fine grain sediment transport (i.e., deposition/ erosion of silt, spread of pollutants trapped over the channel bed or released at the street level in the urban atmospheric boundary layer). We look at the implications for both of these aspects provided by the DNS results.

\section{Coarse Grain Sediment Transport}

Coarse grain sediment transport is directly governed by the drag and lift forces acting on the roughness elements on the bed. These forces can be obtained by integrating shear stress and pressures in the streamwise and vertical directions, respectively, in the turbu-

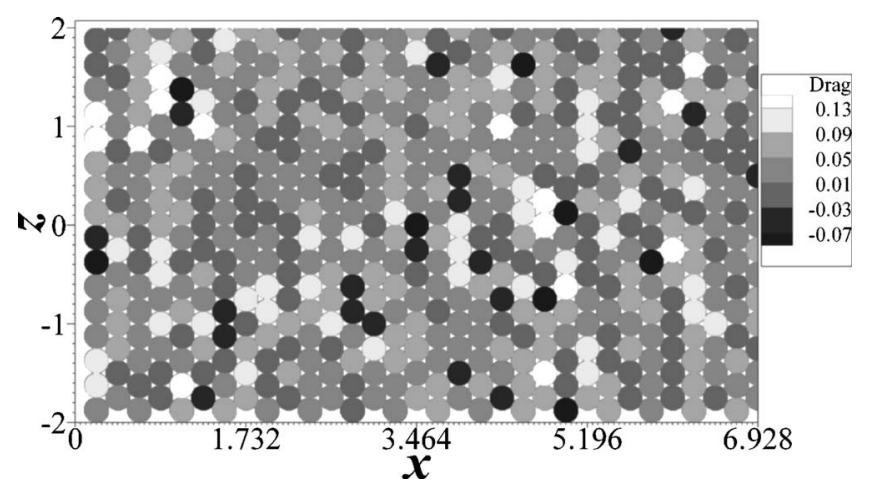

Fig. 18. Distribution of drag on the roughness elements

lent flow field. Both the lift and the drag play a crucial role in the transport of coarse grain sediment. The roughness elements subjected to lift in excess of their weight would be lifted up from the bed and washed away by the fluid. Drag plays an equally important role in uprooting the individual elements through a rolling action in conjunction with a positive vertical lift.

Let us note that a randomly varying distribution of velocity and pressure fields around the roughness elements would give rise to a random distribution of lift and drag experienced by the individual spheres. This is clearly seen in Figs. 17 and 18. There are considerable variations in the lift or drag experienced by individual roughness elements. The lift is positive for the elements which have a low-pressure zone near the top surface (Fig. 19), whereas it is negative for the elements which have a highpressure zone near their top surface (Fig. 20). A probability distribution of the lift in Fig. 21 supports these observations.

Another interesting feature can be observed from Figs. 17 and 18. Many roughness elements which experience very high lift are subjected to high drag as well, and their upstream neighbors experience negative lift. This combination is highly favorable for lifting and rolling of these elements over their upstream neighbors, and thereby disturbing the bed. Whether this would actually happen would need detailed analysis of 3D forces acting on each roughness element. It is useful to note, however, that the uprooting of a roughness element from the bed would require a Shields function $F_{s}=0.056$ (for fully rough flow). This is equivalent to the normalized lift $L \geqslant 0.58$. For the present case, the maximum normalized lift $L_{\text {max }} \approx 0.14$, which indicates that the flow Reynolds number is too low to cause bed instability. At the same time, the preceding analysis provides some insight into the process of possible bed instability at a higher flow Reynolds number as $\mathrm{Wu}$ and Yang (2004) show that near-bed coherent flow structures exhibit

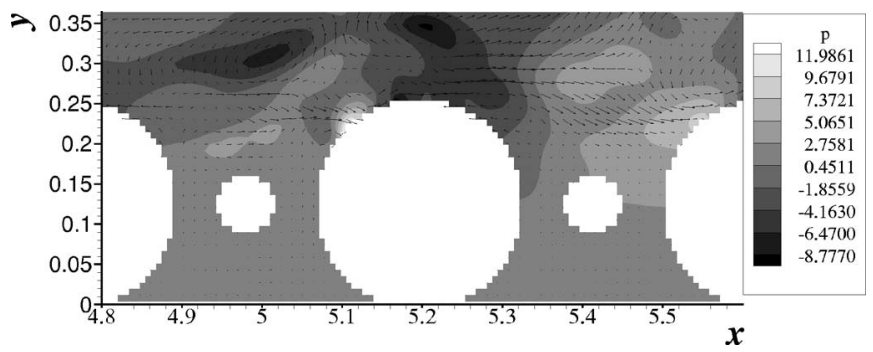

Fig. 19. Velocity vectors in advected frame of reference $(u-\bar{u}, v)$ and contours of pressure on a streamwise-vertical plane through a sphere experiencing positive lift $\left(x_{c} / d=5.2, z_{c} / d=0.012\right)$ 


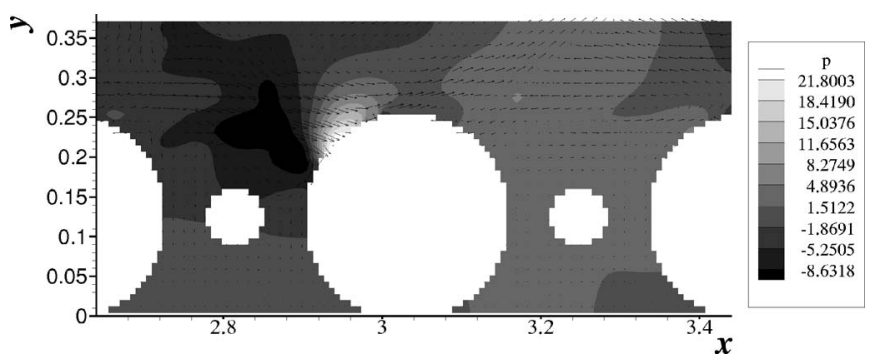

Fig. 20. Velocity vectors in advected frame of reference $(u-\bar{u}, v)$ and contours of pressure on a streamwise-vertical plane through a sphere experiencing negative lift $\left(x_{c} / d=3.04, z_{c} / d=0.012\right)$

consistent correlation patterns which can be characterized by higher-order moments (second, third, and fourth) of velocity fluctuations. They show that these moments are a function of roughness Reynolds number and remain constant for fully rough beds.

\section{Fine Grain Sediment Transport}

Fine grain sediment transport to and from the channel bed depends on the mixing of the fast moving fluid layer above the roughness elements with the apparently quiescent fluid layer between the channel bed and the midplane of the spheres. This mixing is dependent on the vertical components of the fluctuating velocity which are shown in Fig. 22. The presence of both positive and negative values of $v$ indicates good mixing.

A quantitative estimative of the rate of mixing (or exchange) of the bottom layer of the fluid with the fluid above roughness element would be very useful for the transport of fine grain sediments or pollutants on the floor of the channel. Based on the DNS data, we can easily work out the rate of volume exchange and time scales involved. Let $V_{\text {in }}$ and $V_{\text {out }}$ denote the influx and efflux of the fluid from the bottom layer. These are given by

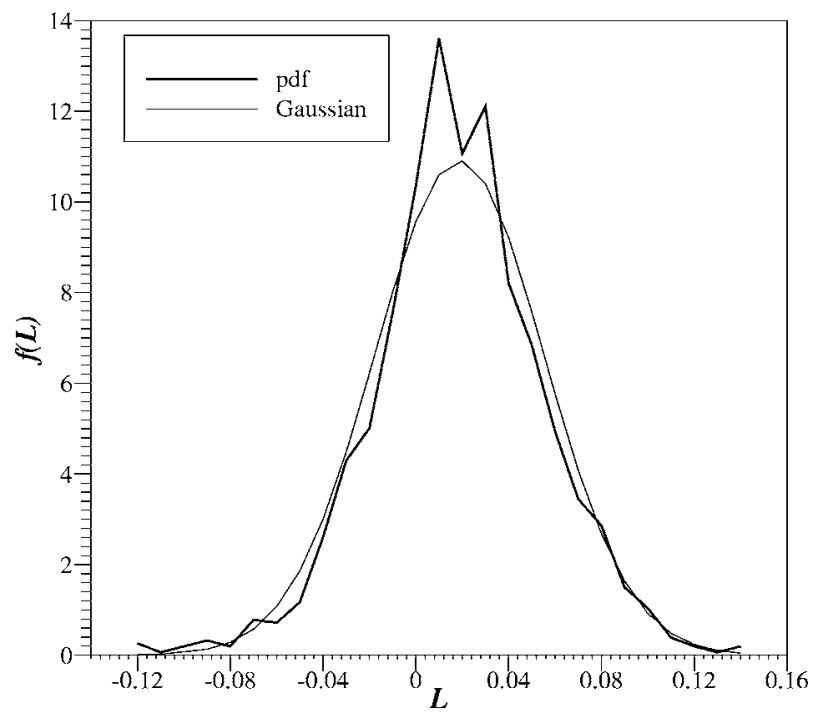

Fig. 21. Probability distribution of the normalized lift experienced by the roughness elements. Lift force has been normalized by $\rho u_{\tau}^{2} d^{2}$.

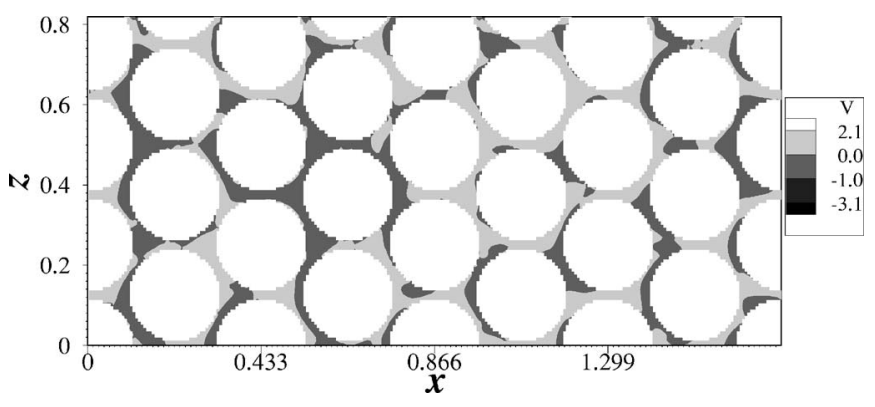

Fig. 22. Contours of vertical velocity at a horizontal plane between the top and midplane of the roughness elements

$$
V_{\text {in }}=\int_{S} \min (v, 0) d x d z, \quad V_{\text {out }}=\int_{S} \max (v, 0) d x d z
$$

where $S=$ horizontal plane passing through the centers of the spheres. Let $V$ be the volume of the fluid contained below the midplane of the spheres. The volume and time exchange coefficients are given by

$$
V_{\text {ex }}=\left|V_{\text {in }}\right| / V, \quad T_{\text {ex }}=V /\left|V_{\text {in }}\right|
$$

$T_{\text {ex }}$ gives the time it would take for nearly complete exchange of the volume $V$ of the fluid in the bottom layer with the fast moving fluid above the roughness elements. Table 2 gives the average values of these quantities. Let us note that the time here is in the units of $d / u_{\tau}$. A vigorous mixing is indicated by the values of the exchange coefficients.

\section{Concluding Remarks}

We have presented a direct numerical simulation of turbulent flow over a rough bed of an open channel consisting of a hexagonal arrangement of the spheres on its bed. The depth of the flow has been taken as four times the diameter of the spheres and the Reynolds number has been chosen so that the roughness Reynolds number is greater than 70, thus ensuring a fully rough flow. A parallel code based on finite difference, domain decomposition, and multigrid methods has been used for the DNS. A detailed analysis of the 3D flow field provided by the DNS has been carried out and the effect of the roughness elements on the flow structures and the implications for sediment transport have been investigated. The conclusions from this investigation are:

1. The first- and second-order statistics obtained from the DNS are (1) in good agreement with experimental results above the roughness layer, and (2) provide accurate supplementary data within the roughness layer where reliable experimental values are very difficult to obtain.

2. The two point correlations and quadrant analysis based on

Table 2. Statistics Related to Exchange Coefficients Based on DNS (Volume $V=1.364$ )

\begin{tabular}{lcccc}
\hline Statistics & $\left|V_{\text {in }}\right|$ & $V_{\text {out }}$ & $V_{\text {ex }}$ & $T_{\text {ex }}$ \\
\hline Mean value & 30.538 & 30.538 & 22.384 & $4.470 \mathrm{E}-02$ \\
Variance & 0.0687 & 0.0687 & 0.0369 & $1.475 \mathrm{E}-06$ \\
Standard deviation & 0.0829 & 0.0829 & 0.0607 & $1.214 \mathrm{E}-03$ \\
Maximum value & 31.722 & 31.722 & 23.254 & $4.633 \mathrm{E}-03$ \\
Minimum value & 29.447 & 29.447 & 21.586 & $4.300 \mathrm{E}-02$ \\
\hline
\end{tabular}


the DNS data confirm the wall similarity hypothesis of Raupach et al. (1991), which states that above the roughness layer, rough bed flow is structurally similar to the smooth bed flow. The DNS results point toward a hierarchy of vortices primarily aligned with the streamwise direction.

3. The DNS data provide detailed information on the flow within the roughness layer (which is very difficult, if not impossible, to obtain experimentally), and clearly indicate vigorous turbulent mixing between the fluid layer trapped below the midplane of the roughness elements and the fast moving fluid above it. Estimates of the time and volume exchange coefficients have been provided which would be relevant to studies of fine grain sediment transport and the dispersal of pollutants trapped on the bed.

4. Spatial and probability distributions of drag and lift experienced by the roughness elements computed from the DNS data provide useful insight into the process of possible bed instability. It is seen that some of the roughness elements which experience very high lift are simultaneously subjected to high drag while their downstream neighbors experience negative lift. This condition is highly favorable for the lifting and rolling of particles over the ones downstream, and thus, disturbing the bed.

\section{Acknowledgments}

This work was carried out under Grant No. GR/M85241 from the U.K. Engineering and Physical Science Research Council (EPSRC). Additional computing resources and support were provided by the U.K. Turbulence Consortium from HPC Grant No. GR/R64957/01. The writers gratefully acknowledge the support of these organizations. Invaluable comments from the reviewers and the Associate Editor for the improvement of the paper are also gratefully acknowledged.

\section{Notation}

The following symbols are used in this paper:

$$
\begin{aligned}
A & =\text { dimensionless constant; } \\
b_{i} & =\text { body force component }(i=x, y, z) ; \\
d & =\text { depth of the channel; } \\
F_{s}= & \text { Shields function; } \\
f(L)= & \text { probability distribution function of lift, } L ; \\
H= & \text { hole size (in quadrant analysis); } \\
h= & \text { effective flow depth }=d-y_{b} ; \\
K= & \text { turbulent kinetic energy; } \\
k= & \text { mean height of roughness elements; } \\
k_{s}= & \text { equivalent sand roughness; } \\
k_{s}^{+}= & \text {roughness Reynolds number; } \\
k_{*}= & \text { measure of the size of roughness elements; } \\
L= & \text { normalized lift on a roughness element; } \\
p= & \text { pressure; } \\
\mathrm{R}_{b}= & \text { Reynolds number based on bulk mean } \\
& \text { velocity }=U_{b} h / \nu ; \\
\mathrm{R}_{c}= & \text { Reynolds number based on top surface } \\
& \text { velocity }=U_{c} h / \nu ; \\
\mathrm{R}_{h}^{+}= & \text {Reynolds number based on bed-shear } \\
R= & \text { velocity }=u_{\tau} h / v ; \\
R_{k}= & \text { correlation coefficient of Reynolds stress; } \\
& \text { turbulent kinetic energy; }
\end{aligned}
$$

$$
\begin{aligned}
& R_{u u}, R_{v v}, R_{w w}=\text { two-point correlations; } \\
& S=\text { horizontal plane through the centers of } \\
& \text { spheres; } \\
& T=\text { large eddy turnover time; } \\
& T_{\text {ex }}=\text { time exchange coefficient; } \\
& t=\text { time; } \\
& U_{b}=\text { bulk mean velocity; } \\
& U_{c}=\text { mean velocity of the top surface; } \\
& U^{+}=\text {normalized temporal and plane average } \\
& \text { streamwise velocity }=\bar{u} / u_{\tau} \text {; } \\
& \bar{u}=\text { temporal and plane averaged streamwise } \\
& \text { velocity at } y \text {; } \\
& u_{i}=\text { velocity component in direction } x_{i} \text {; } \\
& u_{\tau}=\text { bed-shear velocity; } \\
& u, v, w=\text { instantaneous streamwise, vertical, and } \\
& \text { spanwise velocity components; } \\
& u_{\mathrm{rms}}, v_{\mathrm{rms}}, w_{\mathrm{rms}}=\text { turbulence intensities ( } \mathrm{rms} \text { values of } \\
& \text { velocity fluctuations, e.g., } u_{\mathrm{rms}}=\sqrt{\overline{u^{\prime 2}}} \text { ); } \\
& \overline{-u^{\prime} v^{\prime}}=\text { Reynolds stress; } \\
& u^{\prime}, v^{\prime}, w^{\prime}=\text { streamwise, vertical, and spanwise } \\
& \text { turbulent velocity fluctuations (e.g., } \\
& \left.u^{\prime}=u-\bar{u}\right) \\
& V=\text { volume of the fluid contained below the } \\
& \text { midplane of spheres (i.e., the bottom } \\
& \text { layer); } \\
& V_{\mathrm{ex}}=\text { volume exchange coefficient; } \\
& V_{\text {in }}=\text { influx of fluid into the bottom layer; } \\
& V_{\text {out }}=\text { efflux of fluid from the bottom layer; } \\
& x_{i}=\text { Cartesian coordinates }(x, y, z) \text { for } i=1,2,3 \text {; } \\
& x, y, z=\text { streamwise, vertical, and spanwise } \\
& \text { Cartesian coordinates (channel bed is at } \\
& y=0) \text {; } \\
& Y=\text { vertical coordinate with origin at } y=y_{b} \\
& \text { (i.e., } Y=y-y_{b} \text { ); } \\
& Y^{+}=\text {normalized effective height }=Y u_{\tau} / \nu \text {; } \\
& y_{b}=\text { effective location of the bed; } \\
& y_{t}=\text { vertical distance from the top of roughness } \\
& \text { elements; } \\
& y^{+}=\text {normalized hight }=y u_{\tau} / \nu \text {; } \\
& y_{0}=\text { roughness length; } \\
& \Delta x^{+}, \Delta y^{+}, \Delta z^{+}=\text {grid resolution (in wall units); } \\
& \delta=\text { vertical distance of the effective bed from } \\
& \text { the top of roughness elements; } \\
& \delta z=\text { distance to the first minimum of spanwise } \\
& \text { correlation of } u \text {; } \\
& \eta_{i j}=\text { directional dissipation scales of Manhart } \\
& \text { (2000); } \\
& \eta^{+}=\text {Kolmogorov length scale; } \\
& \kappa=\text { von Kármán constant; } \\
& \lambda=\text { mean transverse streak spacing; } \\
& \lambda^{+}=\text {effective viscosity length scale for near } \\
& \text { wall vortical structures }\left(=\lambda u_{\tau} / \nu^{\prime}\right) \text {; } \\
& v=\text { kinematic viscosity of the fluid; } \\
& v^{\prime}=\text { effective kinematic viscosity; and } \\
& \rho=\text { fluid density. }
\end{aligned}
$$

\section{References}

Ashrafian, A., Andersson, H. I., and Manhart, M. (2004). "DNS of turbulent flow in a rod-roughened channel." Int. J. Heat Fluid Flow, 25(3), 373-383.

Bhaganagar, K., and Kim, J. (2002). "Physics of roughwall turbulent 
boundary layer." Bull. Am. Phys. Soc., 47(10), 55-55.

Choi, H., Moin, P., and Kim, J. (1993). "Direct numerical simulation of turbulent flow over riblets." J. Fluid Mech., 255, 503-539.

Defina, A. (1996). "Transverse spacing of low-speed streaks in a channel flow over a rough bed." Coherent flow structures in open channels, P. J. Ashworth, S. J. Bennett, J. L. Best, and S. J. McLelland, eds., Wiley, New York, 87-99.

Grass, A. J. (1971). "Structural features of turbulent flow over smooth and rough boundaries." J. Fluid Mech., 50(2), 233-255.

Grass, A. J., and Mansour-Tehrani, M. (1996). "Generalized scaling of coherent bursting structures in the near-wall region of turbulent flow over smooth and rough boundaries." Coherent flow structures in open channels, P. J. Ashworth, S. J. Bennett, J. L. Best, and S. J. McLelland, eds., Wiley, New York, 41-61.

Grass, A. J., Stuart, R. J., and Mansour-Tehrani, M. (1991). "Vortical structures and coherent motion in turbulent flow over smooth and rough boundaries." Philos. Trans. R. Soc. London, Ser. A, 336(1640), $33-65$.

Kim, J., Moin, P., and Moser, R. (1987). "Turbulence statistics in a fully developed channel flow at low Reynolds number." J. Fluid Mech., 177, 133-166.

Lam, K., and Banerjee, S. (1992). "On the condition of streaks formation in a bounded turbulent flow." Phys. Fluids A, 4(2), 306-320.

Leonardi, S., Orlandi, P., Smalley, R. J., Djenidi, L., and Antonia, R. A. (2003). "Direct numerical simulations of turbulent channel flow with transverse square bars on one wall." J. Fluid Mech., 491, 229-238.

Lu, S. S., and Wilmarth, W. W. (1973). "Measurements of the structure of the Reynolds stress in a turbulent boundary layer." J. Fluid Mech., 60(3), 481-511.

Manhart, M. (2000). "The directional dissipation scale: A criterion for grid resolution in direct numerical simulations." Advances in turbulence VIII, CIMNE, Barcelona, 667-670.

Miyake, Y., Tsujimoto, K., and Agata, Y. (2000). "DNS of a turbulent flow in a rough-wall channel using roughness elements model." JSME Int. J., 43(2), 233-242.

Moin, P., and Mahesh, K. (1998). "Direct numerical simulation: A tool in turbulence research." Annu. Rev. Fluid Mech., 30, 539-578.

Nezu, I. (1977). "Turbulence structure in a open channel flow." Ph.D. thesis, Kyoto Univ., Kyoto, Japan.

Nezu, I., and Nakagawa, H. (1993). Turbulence in open-channel flows, Balkema, Rotterdam, The Netherlands.

Orszag, S. A., and Patterson, G. S. (1972). "Numerical simulation of three-dimensional homogeneous isotropic turbulence." Phys. Rev. Lett., 28(2), 76-79.

Raupach, M. R., Antonia, R. A., and Rajagopalan, S. (1991). "Roughwall turbulent boundary layers." Appl. Mech. Rev., 44(1), 1-25.

Singh, K. M., and Williams, J. J. R. (2005). "A parallel fictitious domain multigrid preconditioner for the solution of Poisson equation in complex geometry." Comput. Methods Appl. Mech. Eng., 194(45-47), $4845-4860$.

Sumitani, Y., and Kasagi, N. (1995). "Direct numerical simulation of turbulent transport with uniform wall suction and injection." AIAA J., 33(7), 1220-1228.

Thomas, T. G., and Williams, J. J. R. (1995). "Turbulent simulation of open channel flow at low Reynolds number." Int. J. Heat Mass Transfer, 38(2), 259-266.

Wu, F.-C., and Yang, K. H. (2004). "Entrainment probabilities of mixedsize sediment incorporating near-bed coherent flow structures." J. Hydraul. Eng., 130(12), 1187-1197. 